\title{
Loops in the Reggeon model for hA scattering
}

\author{
M. Braun, A. Tarasov* \\ Department of High Energy physics, University of S.Petersburg \\ 198904 Ulianovskaya 1, S.Petersburg, Russia
}

\begin{abstract}
Contribution of simplest loops is studied in the Local Reggeon Field Theory with a supercritical pomeron in the nuclear matter. It is shown that the latter transforms the supercritical pomeron into the subcritical. Renormalization of the intercept and difficulties related to the fact that the renormalized intercept is complex are discussed. Numerical results with the conventional parameters are reported.
\end{abstract}

\section{Introduction}

With the advent of QCD much effort has been applied to study high-energy hadron-nucleus scattering within its framework. However QCD can reliably describe only the hard region of the dynamics. Soft processes, contributing to the bulk of the total cross-section, are more difficult to treat. The most sophisticated approach is to study soft processes within the perturbative QCD approach to small $x$ phenomena, based on the Balitsky-Kovchegov equation, which sums all fan diagrams with self-interacting BFKL pomerons [1, 2, 3. However this approach is based on several approximations: a large number of colours $N_{c}$, fixed small QCD coupling constant $\alpha_{s}$ and, most seriously, neglect of loop diagrams. This latter approximation can be justified if the parameter $\gamma=\alpha_{s} \exp \Delta y$ where $y$ is the rapidity and $\Delta$ the pomeron intercept is small. Then for a large nuclear target, such that $A^{1 / 3} \gamma \sim 1$, the tree diagrams indeed give the dominant contribution and loops may be dropped. However with the growth of $y$ the loop contribution becomes not small and although tree diagrams are still relatively enhanced by factor $A^{1 / 3}$, disregard of loops cannot be rigorously justified.

Calculation of the loop contribution within the perturbative QCD appears to be a formidable task. So it seems to be worthwhile to start with a simpler approach, that of the old local Reggeon Field Theory (LRFT) with a supercritical pomeron. Such a study, apart from its possible lessons for the modern QCD approach, also has an independent value, since the old LRFT with phenomenological parameters describes not so badly the soft dynamics of high-energy strong interactions. In fact in many respects it does it better that the perturbative QCD, which encounters severe difficulties due to a very large value of the BFKL intercept, hardly compatible with the experimental data. Also in LRFT applications to hadron-nucleus [4] and nucleus-nucleus [5] collisions were restricted to the approximation, in which the contribution from loop diagrams is neglected. If additionally the slope of the pomeron $\alpha^{\prime}$ is taken to be zero, then the theory is effectively living in zero transverse dimensions and allows for the explicit analytic solution. Still remaining in zero transverse dimension the influence of loop diagrams has also been studied, both theoretically old ago in [6, 7, 8] and numerically recently in [9]. This influence turns out to be decisive for the

*andrey.tarasov@cern.ch 
asymptotic behaviour at large energies and transforms the supercritical pomeron into a weakly subcritical one with the effective intercept $\propto-\exp \left(1 / \lambda^{2}\right)$ where $\lambda$ is the triple-pomeron coupling assumed to be small.

Unfortunately generalization of these beautiful results to the realistic case of two transverse dimensions is prohibitively difficult. To start with, one is forced to introduce a non-zero value of the slope: otherwise the loop contribution is divergent in the impact parameter. However even in the tree approximation solution of the model with $\alpha^{\prime} \neq 0$ is only possible numerically. Second, the model in $d_{T}=2$ needs renormalization in the ultraviolet. And, most important, the method used to solve the model in $d_{T}=0$, which is to study the corresponding quantum-mechanical system and the relevant Schroedinger equation, is inapplicable in the realistic case, since instead of ordinary differential equations one arrives at equations with functional derivatives. In fact summation of all loop contributions is equivalent to a complete solution of the corresponding quantum field theory, the task which seems to be beyond our present possibilities. So at most one can hope to obtain some partial results which might shed light onto the properties of the model with loops. There were several attempts to study the high-energy behaviour of the LRFT with a supercritical pomeron using different approximate techniques and giving contradicting results. In [10] it was claimed that a phase transition occurs at all values of the renormalized intercept $\epsilon^{(r)}=\alpha^{(r)}(0)-1>0$ which leads to a theory which violates the projectile-target symmetry and so physically unacceptable. On the other hand in [11] it was found that the phase transition takes place only at values of $\epsilon^{(r)}$ greater than some critical value $\epsilon_{c}^{(r)}$. At $\epsilon^{(r)}<\epsilon_{c}^{(r)}$ the theory essentially corresponds to that with a subcritical pomeron and cross-sections vanishing at high energies. At $\epsilon^{(r)}>\epsilon_{c}^{(r)}$ crosssections grow as $\log ^{2} s$. No violation of the projectile-target was found. These results were obtained by approximating the transverse plane as a two-dimensional lattice and starting from the known asymptotical solution at each lattice site without intersite interaction. However in this approach transition to the continuous plane requires knowing the single-site solutions at large values of the triple pomeron interaction constant, which are not known.

In the present study we take a different approach. Instead of trying to solve the model for the purely hadronic scattering we consider the hadron-nucleus scattering and propagation of the pomeron inside the heavy nucleus target. Moreover to avoid using numerical solution of the tree diagrams contribution with diffusion in the impact parameter, we concentrate on the case of a constant nuclear density which allows to start with the known analytical solutions. The point which is decisive for the following derivation is that the nuclear surrounding transforms the pomeron from the supercritical one with intercept $\epsilon>0$ to a subcritical one with the intercept $-\epsilon$. Then Regge cuts, corresponding to loop diagrams, start at branch points located to the left of the pomeron pole and their contribution is subdominant at high energies. As a result the theory acquires the properties similar to the standard LRFT with a subcritical pomeron and allows for application of the perturbation theory. For a finite nucleus it leads to cross-sections with tend to a constant value at large energies. For the physical nucleus with a long distance tail it seems to lead to cross-sections growing as $\log ^{2} s$ in agreement with [1].

In fact we cannot prove all these statements rigorously, since we are not able to sum all the loops but only a certain simplest subset of them. However the results we find seem to be rather general.

The paper is organized as follows. In the next section we introduce the model and remind some relevant known results for the zero-slope case $\alpha^{\prime}=0$. In Section 3 we reformulate the model to describe the loops in the nuclear surrounding. Then we calculate the contribution from simplest loops and discuss their renormalization in Section 4. Next in Sections 5 and 6 we sum insertions 
of any number of simplest loops into the scattering amplitude and Green function by solving the relevant Dyson equations. Section 7 gives some numerical illustration of the loop influence for the amplitude and Green function with physically chosen parameters of the theory. Finally Section 8 draws some conclusions.

\section{The model. Zero-slope results}

We study the LRFT model based on two pomeron fields $\phi(y, b)$ and $\phi^{\dagger}(y, b)$ depending on the rapidity $y$ and impact parameter $b$, with a Lagrangian density

$$
L=\phi^{\dagger} S \phi+\lambda \phi^{\dagger} \phi\left(\phi+\phi^{\dagger}\right)+g \rho \phi
$$

Here in the free part

$$
S=\frac{1}{2}\left(\vec{\partial}_{y}-\overleftarrow{\partial_{y}}\right)+\alpha^{\prime} \nabla_{b}^{2}+\epsilon
$$

where $\epsilon$ is the intercept minus unity and $\alpha^{\prime}$ is the slope. The source term describing interaction with the nuclear target is

$$
\rho=A T(b) \delta(y) .
$$

It is assumed that for a supercritical pomeron $\epsilon>0$ and $\lambda<0$. The action is

$$
\mathcal{A}=\int d y \int d^{2} b L(y, b)
$$

and the generating functional is

$$
Z=\int D \phi D \phi^{\dagger} e^{\mathcal{A}}
$$

The free Green function in the momentum space is

$$
G^{(0)}(y, k)=\theta(y) e^{y\left(\epsilon-\alpha^{\prime} k^{2}\right)} .
$$

The classical equation of motion are

$$
\frac{\delta L}{\delta \phi}=-\partial_{y} \phi^{\dagger}+\alpha^{\prime} \nabla_{b}^{2} \phi^{\dagger}+\epsilon \phi^{\dagger}+\lambda \phi^{\dagger^{2}}+2 \lambda \phi \phi^{\dagger}+g \rho=0
$$

and

$$
\frac{\delta L}{\delta \phi^{\dagger}}=\partial_{y} \phi+\alpha^{\prime} \nabla_{b}^{2} \phi+\epsilon \phi+\lambda \phi^{2}+2 \lambda \phi^{\dagger} \phi=0 .
$$

From the latter equation we find $\phi=0$ and the equation for $\phi^{\dagger}$ takes the form

$$
\partial_{y} \phi^{\dagger}=\alpha^{\prime} \nabla_{b}^{2} \phi^{\dagger}+\epsilon \phi^{\dagger}+\lambda \phi^{\dagger^{2}},
$$

with an initial condition

$$
\phi^{\dagger}(y=0)=g A T(b) .
$$

Equation (9) describes evolution of the pomeron field in rapidity and its diffusion in the impact parameter inside the nucleus. In the approximation $\alpha^{\prime}=0$ equation for $\phi^{\dagger}$ simplifies to

$$
\partial_{y} \phi^{\dagger}=\epsilon \phi^{\dagger}+\lambda \phi^{\dagger^{2}}
$$


which is trivially solved for each given $b$ [4]:

$$
\phi^{\dagger}(y, b)=\frac{g A T(b) e^{\epsilon y}}{1-\lambda g A T(b) \frac{1}{\epsilon}\left(e^{\epsilon y}-1\right)} \equiv \xi(y, b) .
$$

The scattering matrix is

$$
T(y, b)=g \xi(y, b)
$$

\section{$3 \quad$ Loops in the nuclear surrounding}

\subsection{Transformation to the nuclear background}

In this section we shall analyze the structure of the model beyond the tree approximation in the nuclear background. To locate loops we make a shift in field $\phi^{\dagger}$ :

$$
\phi^{\dagger}(y, b)=\phi_{1}^{\dagger}(y, b)+\xi(y, b)
$$

and reinterpret our theory in terms of fields $\phi$ and $\phi_{1}^{\dagger}$.

The Lagrangian density becomes

$$
L=\left(\phi_{1}^{\dagger}+\xi\right) S \phi+\lambda\left(\phi_{1}^{\dagger}+\xi\right)^{2} \phi+\lambda \phi^{2}\left(\phi_{1}^{\dagger}+\xi\right)+g \rho \phi .
$$

Terms linear in $\phi$ vanish due to equation of motion for $\xi$. We are left with

$$
L=\phi_{1}^{\dagger}(S+2 \lambda \xi) \phi+\lambda \xi \phi^{2}+\lambda \phi_{1}^{\dagger} \phi\left(\phi_{1}^{\dagger}+\phi\right) .
$$

This Lagrangian corresponds to a theory in the vacuum with the pomeron propagator in the external field $f(b, y)=2 \lambda \xi(y, b)$

$$
G_{f}^{(0)}=-(S+2 \lambda \xi)^{-1}
$$

the standard triple interaction and an extra interaction described by the term $\lambda \xi \phi^{2}$. This new interaction corresponds to the transition of a pair of pomerons into the vacuum at point $(y, b)$ with a vertex $\lambda \xi(y, b)$, see Fig. 1 .

Loops in the Green function may be formed both by the standard interaction and the new one. In the latter case they are to be accompanied by at least a pair of of standard interactions. Diagrams with a few simple loops in the Green function are illustrated in Fig. 2. One immediately observes that a loop formed by the standard interaction has the order $\lambda^{2} / \alpha^{\prime}$ and requires renormalization. A loop formed by a new interaction has the order $\lambda^{3} / \alpha^{\prime}$ and is finite.

The amplitude is obtained as a tadpole $\left.g<\phi_{1}^{\dagger}(y, b)\right\rangle$. The simplest diagrams for it contains one loop and are shown in Fig. $3 a, b$. Diagrams with more loops are shown in Figs. $3 c, d$.

\subsection{The Green function in the external field $2 \lambda \phi^{\dagger}(y)$}

In the construction of loops there appears a Green function $G_{f}^{(0)}\left(y, b \mid y^{\prime} b^{\prime}\right)$ in which the pomeron fields are coupled to the external field

$$
f(y, b)=2 \lambda \xi(y, b) .
$$


This Green function satisfies the equation

$$
\frac{d G_{f}^{(0)}\left(y, b \mid y^{\prime} b^{\prime}\right)}{d y}=\left(\epsilon+\alpha^{\prime} \nabla_{b}^{2}\right) G_{f}^{(0)}\left(y, b \mid y^{\prime}, b^{\prime}\right)+f(y, b) G_{f}^{(0)}\left(y, b \mid y^{\prime}, b^{\prime}\right)
$$

with the boundary conditions

$$
G_{f}^{(0)}\left(y, b \mid y^{\prime}, b^{\prime}\right)=0, \quad y-y^{\prime}<0, \quad G^{(0)}\left(y^{\prime}, b \mid y^{\prime}, b^{\prime}\right)=\delta^{2}\left(b-b^{\prime}\right) .
$$

In the general case the Green function $G_{f}^{(0)}$ can only be calculated numerically, just as the external field $f(y, b)$. Its analytic form can be found in two cases.

If the slope $\alpha^{\prime}=0$ then obviously we have

$$
G_{f}^{(0)}\left(y, b \mid y^{\prime}, b^{\prime}\right)=\delta^{2}\left(b-b^{\prime}\right) G_{f}^{(0)}\left(y, y^{\prime}, b\right),
$$

where $G_{f}^{(0)}\left(y, y^{\prime}, b\right)$ is the Green function in the zero-dimensional world which satisfies

$$
\frac{d G_{f}^{(0)}\left(y, y^{\prime}, b\right)}{d y}=\epsilon G_{f}^{(0)}\left(y, y^{\prime}, b\right)+f(y, b) G_{f}^{(0)}\left(y, y^{\prime}, b\right)
$$

with the boundary conditions

$$
G_{f}^{(0)}\left(y, y^{\prime}, b\right)=0, \quad y-y^{\prime}<0, \quad G_{f}^{(0)}\left(y^{\prime}, y^{\prime}, b\right)=1
$$

and $f(y, b)$ given by (18) and (12). Solution of (22) for $G_{f}^{(0)}$ is trivial. It is easy to find that

$$
G_{f}^{(0)}\left(y, y^{\prime}, b\right)=e^{\int_{y^{\prime}}^{y} d s(\epsilon+f(s, b))}=e^{-\epsilon\left(y-y^{\prime}\right)}\left(\frac{a-(a-1) e^{-\epsilon y^{\prime}}}{a-(a-1) e^{-\epsilon y}}\right)^{2}=e^{\epsilon\left(y-y^{\prime}\right)} \frac{p^{2}\left(y^{\prime}\right)}{p^{2}(y)},
$$

where

$$
a=-\frac{\lambda g A T(b)}{\epsilon}>0
$$

and

$$
p(y)=1+a\left(e^{\epsilon y}-1\right)
$$

It is remarkable that at large $y$ the Green function $G_{f}^{(0)}\left(y, y^{\prime}, b\right) \simeq \exp (-\epsilon y)$, that is behaves as the free Green function with the opposite sign of $\epsilon$ and so vanishes at $y \rightarrow \infty$. In contrast to the free Green function, it corresponds to a subcritical pomeron.

The second case which admits an analytic solution for the Green function is that of the nuclear matter, that is the case when the profile function is constant in all transverse space

$$
T(b)=T_{0} .
$$

Physically this case corresponds to the behaviour of the Green function at values of of the impact parameter well inside the nucleus, where the variation of $T(b)$ is small. Then the classical field $\xi$ and the external field $f=2 \lambda \xi$ become $b$-independent and the equation for the Green function in this field takes the form

$$
\frac{d G_{f}^{(0)}\left(y, b \mid y^{\prime}, b^{\prime}\right)}{d y}=\left(\epsilon+\alpha^{\prime} \nabla_{b}^{2}\right) G_{f}^{(0)}\left(y, b \mid y^{\prime}, b^{\prime}\right)+f(y) G_{f}^{(0)}\left(y, b \mid y^{\prime}, b^{\prime}\right)
$$


with the same initial condition (20). This equation can also be easily solved. We present

$$
G_{f}^{(0)}\left(y, b \mid y^{\prime}, b^{\prime}\right)=X\left(y-y^{\prime}, b, b^{\prime}\right) e^{\int_{y^{\prime}}^{y} d y_{1}\left(\epsilon+f\left(y_{1}\right)\right)}=X\left(y-y^{\prime}, b, b^{\prime}\right) G_{f}^{(0)}\left(y, y^{\prime}, b\right),
$$

where $G_{f}^{(0)}\left(y, y^{\prime}, b\right)$ is the Green function in zero-dimensional world (24) satisfying $G_{f}^{(0)}\left(y^{\prime}, y^{\prime}, b\right)=1$ Then we obtain an equation for $X$ :

$$
\partial_{y} X\left(y, b, b^{\prime}\right)=\alpha^{\prime} \nabla_{b}^{2} X_{f}\left(y, b, b^{\prime}\right)
$$

with an initial condition

$$
X\left(0, b, b^{\prime}\right)=\delta^{2}\left(b-b^{\prime}\right)
$$

and thus with the solution

$$
X\left(y-y^{\prime}, b, b^{\prime}\right)=\frac{1}{4 \pi \alpha^{\prime}\left(y-y^{\prime}\right)} e^{-\left(b-b^{\prime}\right)^{2} /\left(4 \alpha^{\prime}\left(y-y^{\prime}\right)\right)} .
$$

So in the nuclear matter the Green function in the external field takes a simple form

$$
G_{f}^{(0)}\left(y, b \mid y^{\prime}, b^{\prime}\right)=\frac{1}{4 \pi \alpha^{\prime}\left(y-y^{\prime}\right)} e^{-\epsilon\left(y-y^{\prime}\right)-\left(b-b^{\prime}\right)^{2} /\left(4 \alpha^{\prime}\left(y-y^{\prime}\right)\right)}\left(\frac{a-(a-1) e^{-\epsilon y^{\prime}}}{a-(a-1) e^{-\epsilon y}}\right)^{2},
$$

where we used (24). In the momentum space we find

$$
G_{f}^{(0)}\left(y, y^{\prime}, k\right)=e^{-\left(y-y^{\prime}\right)\left(\epsilon+\alpha^{\prime} k^{2}\right)}\left(\frac{a-(a-1) e^{-\epsilon y^{\prime}}}{a-(a-1) e^{-\epsilon y}}\right)^{2}=e^{\left(y-y^{\prime}\right)\left(\epsilon-\alpha^{\prime} k^{2}\right)} \frac{p^{2}\left(y^{\prime}\right)}{p^{2}(y)} .
$$

Note an especially simple case when $a=1$ and the Green function in the external field coincides with the free Green function with the opposite sign of $\epsilon$ :

$$
\left.G_{f}^{(0)}\left(y, y^{\prime}, k\right)\right|_{a=1}=e^{-\left(y-y^{\prime}\right)\left(\epsilon+\alpha^{\prime} k^{2}\right)} .
$$

Then the theory formally corresponds to a subcritical pomeron model with an additional interaction shown in Fig. 1

\section{Lowest order loops}

\subsection{The lowest order loop in absence of the nucleus and its renormalization}

Let us study the simplest contribution from loops, the single loop in the Green function. In the second order in $\lambda$ we get for the Green function

$$
G^{(2)}(y, k)=-2 \lambda^{2} \int d y_{1} d y_{2} \frac{d^{2} k_{1}}{(2 \pi)^{2}} G^{(0)}\left(y-y_{1}, k\right) G^{(0)}\left(y_{1}-y_{2}, k_{1}\right) G^{(0)}\left(y_{1}-y_{2}, k-k_{1}\right) G^{(0)}\left(y_{2}, k\right) .
$$

The minus sign comes from the fact that the simple Green function is in fact $-G^{(0)}$, so that in the second order we get $-G^{(0)} *\left(-G^{(0)}{ }^{2}\right)\left(-G^{(0)}\right)=-G^{(2)}$. Factor 2 comes from the contraction of $\phi^{\dagger^{2}}\left(y_{1}, b_{1}\right) \phi^{2}\left(y_{2}, b_{2}\right)$. Integration over $k_{1}$ is done trivially

$$
\Sigma(y, k) \equiv-2 \lambda^{2} \int \frac{d^{2} k_{1}}{(2 \pi)^{2}} G^{(0)}\left(y, k_{1}\right) G^{(0)}\left(y, k-k_{1}\right)=-C \frac{1}{y} e^{2 \epsilon y-(1 / 2) \alpha^{\prime} y k^{2}},
$$


where

$$
C=\frac{\lambda^{2}}{4 \pi \alpha^{\prime}} .
$$

$\Sigma(y, k)$ is finite as it stands but leads to divergence at $y=0$ when substituted into (36). So it requires renormalization, which is achieved by introducing an extra mass term $\Delta \epsilon \phi^{\dagger} \phi$ into the Lagrangian. The renormalized self-mass is therefore

$$
\Sigma^{(r)}(y, k)=\Sigma(y, k)+\Delta \epsilon \delta(y)
$$

and we have to require that

$$
\int_{0}^{y} d y^{\prime} \Sigma^{(r)}\left(y^{\prime}, k\right)=\text { finite terms }-C \ln \frac{1}{y_{\min }}+\Delta \epsilon<\infty,
$$

So we conclude that

$$
\Delta \epsilon=-C \ln \left(c_{R} y_{\min }\right),
$$

where $y_{\text {min }}$ is the cutoff at small values of $y$ and $c_{R}$ is an arbitrary finite constant. Thus we find that the renormalized self mass is

$$
\Sigma^{(r)}(y, k)=-C\left(\frac{1}{y} e^{2 \epsilon y-(1 / 2) \alpha^{\prime} y k^{2}}+\delta(y) \ln \left(c_{R} y_{\min }\right)\right) .
$$

or, in the impact parameter space,

$$
\Sigma^{(r)}(y, b)=-C\left(\frac{1}{2 \pi \alpha^{\prime} y^{2}} e^{2 \epsilon y-b^{2} /\left(2 \alpha^{\prime} y\right)}+\delta(y) \delta^{2}(b) \ln \left(c_{R} y_{\min }\right)\right) .
$$

The Dyson equation for the Green function becomes

$$
G(y, 0, k)=G^{(0)}(y, 0, k)+\int_{0}^{y} d y_{1} G^{(0)}\left(y-y_{1}, k\right) \int_{0}^{y_{1}} d y_{2} \Sigma^{(r)}\left(y_{1}-y_{2}, k\right) G\left(y_{2}, k\right) .
$$

It is trivially solved by the Laplace transformation. We introduce Laplace transforms

$$
G(E, k)=\int_{0}^{\infty} d y e^{-E y} G(y, 0, k)
$$

and similarly $G^{(0)}(E, k)$ and $\Sigma^{(r)}(E, k)$. In terms of the Laplace transforms

$$
G(E, k)=\frac{1}{\left(G^{(0)}(E, k)\right)^{-1}-\Sigma^{(r)}(E, k)}
$$

From (6) and (42) we have

$$
G^{(0)}(E, k)=\frac{1}{E-\epsilon+\alpha^{\prime} k^{2}}
$$

and

$$
\begin{gathered}
\Sigma^{(r)}(E, k)=-C\left(\int_{y_{\min }}^{\infty} \frac{d y}{y} e^{-y\left(E-2 \epsilon+\alpha^{\prime} k^{2} / 2\right)}+\ln \left(c_{R} y_{\min }\right)\right) \\
=C\left(\ln \left(E-2 \epsilon+\alpha^{\prime} k^{2} / 2\right)+C_{E}-\ln c_{R}\right) .
\end{gathered}
$$


(Note that terms with $\ln \left(y_{\min }\right)$ cancel). So the Laplace transform for the Green function is

$$
G^{-1}(E, k)=E-\epsilon+\alpha^{\prime} k^{2}-C\left[\ln \left(E-2 \epsilon+\alpha^{\prime} k^{2} / 2\right)+C_{E}-\ln c_{R}\right] .
$$

Its singularities in the complex $E$-plane consist of a branchpoint at $E=2 \epsilon-\alpha^{\prime} k^{2} / 2$ and a pole $E_{0}(k)$ determined by the equation:

$$
G^{-1}\left(E_{0}, k\right)=0
$$

At small $\lambda$ Eq. (50) gives two complex conjugate poles

$$
E_{0}^{( \pm)}=\epsilon-\alpha^{\prime} k^{2}+C\left(\ln \left(\left|\epsilon+\alpha^{\prime} k^{2} / 2\right|\right)+C_{E}-\ln c_{R} \pm i \pi\right)
$$

These poles appear on the physical sheet due to the abnormal sign of the self-mass contribution, which is a consequence of the imaginary coupling in the original Lagrangian of the LRFT. The asymptotic of the Green function is $\sim \exp (2 \mu y)$ in accordance of the contribution of the Regge cut for a supercritical pomeron. According to (41) the unrenormalized pomeron intercept (depending on the cutoff) is

$$
\epsilon_{0}\left(y_{0}\right)=\epsilon-C \ln \left(c_{R} y_{\min }\right) .
$$

It goes to infinity as $y_{\min } \rightarrow 0$, since $C>0$. As to the renormalized intercept its definition may be chosen in different ways. Traditionally it may be taken as the position of the pole in $E$ in the Green function at $k=0$. Then it is given by (50), complex and $c_{R}$-dependent. One observes the difficulty in doing renormalization in the standard way by requiring the zero order propagator to have the pole at a chosen energy $E_{0}(k=0)$ : unless $E_{0}>2 \epsilon$ there are two complex conjugate values of $E_{0}$ and if one chooses $E_{0}>2 \epsilon$ contributions from higher order self mass will again make it complex. So the structure of perturbative singularities prohibits choosing the zero-order propagator to carry the pole singularity, since there are two. However from the physical point of view such a choice is not necessary Inclusion of more pomeron exchanges shifts the dominating singularity further to the right, so that the simple pole contribution to any physical process becomes completely obliterated. It is more reasonable to relate the value of the renormalized intercept (and thus fix the arbitrary constant $c_{R}$ ) to some physical observables. In the following we shall see that in the scattering on the nucleus there appears a possibility to define the renormalized intercept from experimental data at high energies, with a certain choice of $c_{R}$.

\subsection{Lowest order loop in the nuclear matter}

The contribution from the lowest order loop (Fig. $2 a$ ) in the nuclear matter to the Green function in is

$$
G_{f}^{(2)}(y, 0, k)=\int d y_{1} d y_{2} G_{f}^{(0)}\left(y, y_{1}, k\right) \Sigma_{1}\left(y_{1}, y_{2}, k\right) G_{f}^{(0)}\left(y_{2}, 0, k\right)
$$

where the Green functions $G_{f}^{(0)}$ are given by $(\underline{24})$ and

$$
\Sigma_{1}\left(y_{1}, y_{2}, k\right)=-C\left(\frac{1}{y_{1}-y_{2}} e^{-2 \epsilon\left(y_{1}-y_{2}\right)-(1 / 2)\left(y_{1}-y_{2}\right) \alpha^{\prime} k^{2}} e^{2 \int_{y_{2}}^{y_{1}} d s f(s)}+\delta\left(y_{1}-y_{2}\right) \ln \left(c_{R} y_{\min }\right)\right) .
$$

is the renormalized 2nd order self mass in the nuclear background. The subtraction term is easily calculated:

$$
G_{f, \text { subtr }}^{(2)}=-C \ln \left(c_{R} y_{\text {min }}\right) \int d y_{1} G_{f}^{(0)}\left(y, y_{1}, k\right) G_{f}^{(0)}\left(y_{1}, 0, k\right)=-C \ln \left(c_{R} y_{\text {min }}\right) y G_{f}^{(0)}(y, 0, k) .
$$


The main term is given by

$$
-C G_{f}^{(0)}(y, 0, k) \int_{0}^{y} d y_{1} \int_{0}^{y_{1}} d y_{2} \frac{1}{y_{1}-y_{2}} e^{\left(y_{1}-y_{2}\right)\left(\epsilon+\frac{1}{2} \alpha^{\prime} k^{2}\right)} \frac{p^{2}\left(y_{2}\right)}{p^{2}\left(y_{1}\right)} .
$$

Changing to integration variable $z=y_{1}-y_{2}$ we find

$$
G_{f, \text { main }}^{(2)}(y, 0, k)=-C G_{f}^{(0)}(y, 0, k) \int_{0}^{y} \frac{d z}{z} W(z)
$$

where

$$
W(z)=e^{z\left(\epsilon+\frac{1}{2} \alpha^{\prime} k^{2}\right)} \int_{z}^{y} d y_{1} \frac{p^{2}\left(y_{1}-z\right)}{p^{2}\left(y_{1}\right)} \equiv e^{\frac{1}{2} z \alpha^{\prime} k^{2}} \tilde{W}(z)
$$

We note that $W(0)=y$ so that we can write

$$
G_{f, \text { main }}^{(2)}(y, 0, k)=-C G_{f}^{(0)}(y, 0, k)\left\{\int_{0}^{y} \frac{d z}{z}(W(z)-y)+y \ln \frac{y}{y_{\min }}\right\} .
$$

The second term just substitutes in the subtraction contribution $y_{\min }$ by $y$. The first term can be integrated numerically since it converges at $z=0$.

Function $\tilde{W}(z)$ can be calculated analytically. Passing to integration variable $u=e^{\epsilon y_{1}}$ and doing simple integrals we find

$$
\tilde{W}(z)=e^{-\epsilon z}\left(y-z+\frac{q_{1}^{2}-q^{2}}{\epsilon q^{2}} \ln \frac{u_{2}\left(u_{1}+q\right)}{u_{1}\left(u_{2}+q\right)}-\frac{\left(q_{1}-q\right)^{2}}{\epsilon q} \frac{u_{2}-u_{1}}{\left(u_{2}+q\right)\left(u_{1}+q\right)}\right) .
$$

where $u_{1}=e^{\epsilon z}, u_{2}=e^{\epsilon y}, q=1 / a-1$ and $q_{1}=q u_{1}$.

Note that after renormalization the 2 nd order contribution to the Green function is also finite in the nuclear background.

\subsection{3d order loop Fig. $2 b$}

The new loop shown in Fig. $2 b$, which appears in the nucleus, has formally order $\lambda^{3}$. However it is proportional to $A T(b)$, so that the extra $\lambda$ combines into the characteristic parameter $a$, which is not small. So in fact it has the same order of magnitude as the simple loop of Fig. $2 a$.

The contribution to the pomeron self-mass corresponding to Fig. $2 b$ is

$$
\Sigma_{2}\left(y_{2}, y_{3}, k\right)=4 \lambda^{3} \int_{0}^{y_{3}} d y_{1} \int \frac{d^{2} k_{1}}{(2 \pi)^{2}} G_{f}^{(0)}\left(y_{2}, y_{3}, k-k_{1}\right) G_{f}^{(0)}\left(y_{2}, y_{1}, k_{1}\right) G_{f}^{(0)}\left(y_{3}, y_{1}, k_{1}\right) \xi\left(y_{1}\right) .
$$

Here it is assumed that $y_{2}>y_{3}$. The final factor 4 combines factor 4 from contractions of $\phi^{2}\left(y_{1}\right)$ with $\phi^{\dagger^{2}}\left(y_{2}\right)$ and $\phi^{\dagger^{2}}\left(y_{3}\right)$, factor 2 coming from interchange $2 \leftrightarrow 3$ and factor $1 / 2$ left from $1 / 3$ ! in the development of action in the third order in interaction. The sign "+" comes from the odd number of propagators in the contribution to the full propagator with a minus sign. Using explicit expressions for the propagators and $\xi$ and definition (25) we rewrite (61) as

$$
\Sigma_{2}\left(y_{2}, y_{3}, k\right)=-4 \lambda^{2} a \epsilon \int_{0}^{y_{3}} d y_{1} \frac{p^{3}\left(y_{1}\right)}{p^{4}\left(y_{2}\right)} e^{\epsilon\left(2 y_{2}-y_{1}\right)-\left(y_{2}-y_{3}\right) \alpha^{\prime} k^{2}} \int \frac{d^{2} k_{1}}{(2 \pi)^{2}} e^{-2 \alpha^{\prime} k_{1}^{2}\left(y_{2}-y_{1}\right)+2\left(y_{2}-y_{3}\right) \alpha^{\prime} k k_{1}} .
$$


The integral over $k_{1}$ is

$$
\frac{1}{8 \pi \alpha^{\prime}\left(y_{2}-y_{1}\right)} e^{\alpha^{\prime} k^{2} \frac{\left(y_{2}-y_{3}\right)^{2}}{2\left(y_{2}-y_{1}\right)}}
$$

so that in the end we obtain

$$
\Sigma_{2}\left(y_{2}, y_{3}, k\right)=-\frac{\lambda^{2} a \epsilon}{2 \pi \alpha^{\prime}} \frac{1}{p^{4}\left(y_{2}\right)} e^{2 \epsilon y_{2}-\left(y_{2}-y_{3}\right) \alpha^{\prime} k^{2}} \int_{0}^{y_{3}} \frac{d y_{1}}{y_{2}-y_{1}} p^{3}\left(y_{1}\right) e^{-\epsilon y_{1}+\alpha^{\prime} k^{2} \frac{\left(y_{2}-y_{3}\right)^{2}}{2\left(y_{2}-y_{1}\right)}}
$$

It is trivial to see that $\Sigma_{2}$ is finite and does not need any more renormalization.

\section{Amplitude in the nuclear matter approximation}

\subsection{Lowest order}

We start from the lowest order approximation, when at fixed $b$ the scattering amplitude is

$$
T^{(0)}(y, b)=g^{2} A T(b) \frac{e^{\epsilon y}}{p(y)} .
$$

The total forward scattering amplitude is obtained after integration over all $b$ :

$$
T^{(0)}(y)=\int d^{2} b T^{(0)}(y, b) .
$$

This expression can be rewritten in the momentum space, once we introduce the Fourier transform of $T(y, b)$ by

$$
T^{(0)}(y, b)=\int \frac{d^{2} k}{(2 \pi)^{2}} e^{i k b} \tilde{T}^{(0)}(y, k)
$$

as

$$
T^{(0)}(y)=\int \frac{d^{2} b d^{2} k}{(2 \pi)^{2}} e^{i k b} \tilde{T}^{(0)}(y, k)=\tilde{T}^{(0)}(y, 0) .
$$

In the nuclear matter $T(y, b)$ does not depend on $b$ :

$$
T^{(0)}(y, b)=g^{2} A T_{0} \frac{e^{\epsilon y}}{p(y)},
$$

so that its Fourier transform is

$$
\tilde{T}^{(0)}(y, k)=(2 \pi)^{2} \delta^{2}(k) g^{2} A T_{0} \frac{e^{\epsilon y}}{p(y)} .
$$

From (65) the forward scattering amplitude is

$$
T^{(0)}(y)=\int d^{2} b t(y)=\pi R_{A}^{2} g^{2} A T_{0} \frac{e^{\epsilon y}}{p(y)}=g^{2} A \frac{e^{\epsilon y}}{p(y)},
$$

where we used that $T_{0}=1 /\left(\pi R_{A}^{2}\right)$. Due to (67) this of course implies that $(2 \pi)^{2} \delta^{2}(k=0) \rightarrow \pi R_{A}^{2}$. In the limit $y \rightarrow \infty$ the lowest order amplitude tends to a finite value

$$
T^{(0)}(y)_{y \rightarrow \infty}=\frac{g^{2} A}{a}=\pi R_{A}^{2} \frac{g \epsilon}{|\lambda|} .
$$




\subsection{2nd order}

In the next order we have

$$
\begin{aligned}
& \tilde{T}^{(2)}(y, k)=\int d y_{1} d y_{2} G_{f}^{(0)}\left(y, y_{1}, k\right) \Sigma_{1}\left(y_{1}, y_{2}, k\right) \tilde{T}^{(0)}\left(y_{2}, k\right) \\
& =(2 \pi)^{2} \delta^{2}(k) g^{2} A T_{0} \int d y_{1} d y_{2} G_{f}^{(0)}\left(y, y_{1}, 0\right) \Sigma_{1}\left(y_{1}, y_{2}, 0\right) \frac{e^{\epsilon y_{2}}}{p\left(y_{2}\right)}
\end{aligned}
$$

so that the forward scattering amplitude is

$$
T^{(2)}(y)=\pi R_{A}^{2} \int d y_{1} d y_{2} G_{f}\left(y, y_{1}, 0\right) \Sigma_{1}\left(y_{1}, y_{2}, 0\right) T^{(0)}\left(y_{2}, b\right) .
$$

The subtraction term in $\Sigma^{(r)}$ gives a contribution

$$
T_{s u b}^{(2)}(y)=-C \pi R_{A}^{2} \ln \left(c_{R} y_{\min }\right) \int_{0}^{y} d y_{1} G_{f}^{(0)}\left(y, y_{1}, 0\right) T^{(0)}\left(y_{1}, b\right) .
$$

Using (68) and (24) we get

$$
T_{\text {sub }}^{(2)}(y)=-C g^{2} A G_{f}^{(0)}(y, 0,0) \ln \left(c_{R} y_{\min }\right) \int_{0}^{y} d y_{1} p\left(y_{1}\right) .
$$

We define

$$
I(y, z) \equiv \int_{z}^{y} d y_{1} p\left(y_{1}\right)=\frac{a}{\epsilon}\left(e^{\epsilon y}-e^{\epsilon z}\right)+(y-z)(1-a) .
$$

Then we find

$$
T_{s u b}^{(2)}(y)=-C g^{2} A \frac{e^{\epsilon y}}{p^{2}(y)} \ln \left(c_{R} y_{\min }\right) I(y, 0) .
$$

The main term is

$$
T_{\text {main }}^{(2)}(y)=-C g^{2} A \frac{e^{\epsilon y}}{p^{2}(y)} \int_{0}^{y} d y_{1} \int_{0}^{y_{1}} d y_{2} e^{\epsilon\left(y_{1}-y_{2}\right)} \frac{1}{y_{1}-y_{2}} \frac{p^{3}\left(y_{2}\right)}{p^{2}\left(y_{1}\right)} .
$$

Passing to integration variables $y_{1}$ and $z=y_{1}-y_{2}$ we find

$$
T_{\text {main }}^{(2)}(y)=-C g^{2} A \frac{e^{\epsilon y}}{p^{2}(y)} \int_{0}^{y} \frac{d z}{z} \Psi(z),
$$

where

$$
\Psi(z)=e^{\epsilon z} \int_{z}^{y} d y_{1} \frac{p^{3}\left(y_{1}-z\right)}{p^{2}\left(y_{1}\right)} .
$$

Note that

$$
\Psi(0)=I(y, 0)
$$

Thus we find

$$
T_{\text {main }}^{(2)}(y)=-C g^{2} A \frac{e^{\epsilon y}}{p^{2}(y)}\left\{\int_{0}^{y} \frac{d z}{z}(\Psi(z)-\Psi(0))+\ln \frac{y}{y_{\min }} I(y, 0)\right\} .
$$

The second term just changes $y_{\min }$ to $y$ in the subtraction term. 
finds

Function $\Psi(z)$ can be calculated analytically in the same way as $\tilde{W}(z)$ (see Eq. (58)). One

$$
\begin{gathered}
\Psi(z)=\frac{I(y, z)}{u_{1}^{2}}+3 a q \frac{u_{1}-1}{u_{1}^{2}}(y-z)+a q\left(2+u_{1}\right) \frac{\left(u_{1}-1\right)^{2}}{\epsilon u_{1}^{2}} \ln \frac{u_{2}\left(u_{1}+q\right)}{u_{1}\left(u_{2}+q\right)} \\
+a q^{2} \frac{\left(u_{1}-1\right)^{3}}{\epsilon u_{1}^{2}} \frac{u_{1}-u_{2}}{\left(u_{2}+q\right)\left(u_{1}+q\right)} .
\end{gathered}
$$

where as before $u_{1}=e^{\epsilon z}, u_{2}=e^{\epsilon y}, q=1 / a-1$ and $q_{1}=q u_{1}$. The remaining integration over $z$ has to be done numerically.

It is not difficult to find the asymptotic behaviour of $T^{(2)}(y)$ at large $y$. At $y \rightarrow \infty$, asymptotically

$$
p(y)=a e^{\epsilon y}, \quad I(y, 0)=\frac{a}{\epsilon} e^{\epsilon y}
$$

so that in this limit

$$
T^{(2)}(y)_{s u b}=-C \frac{g^{2} A}{a \epsilon} \ln \left(c_{R} y_{\min }\right)
$$

The asymptotic of the main term comes from large values of $z$ inside the integral. At large $z$

$$
\Psi(z)-\Psi(0)=-\frac{a}{\epsilon} e^{\epsilon y}\left(1-e^{-2 \epsilon z}\right)
$$

and so

$$
\int^{y} \frac{d z}{z}(\Psi(z)-\Psi(0)) \simeq \frac{a}{\epsilon} e^{\epsilon y}\left(\operatorname{Ei}(-2 \epsilon y)-\ln (2 \epsilon y)-C_{E}\right) \simeq-\frac{a}{\epsilon} e^{\epsilon y}\left(\ln (2 \epsilon y)+C_{E}\right) .
$$

Thus asymptotically

$$
T_{\text {main }}^{(2)}(y)=C \frac{g^{2} A}{a \epsilon}\left(\ln \left(2 \epsilon y_{\min }\right)+C_{E}\right) .
$$

In the sum the terms with $\ln y_{\min }$ cancel and we find that in the limit $y \rightarrow \infty$ the loop contribution also tends to a constant

$$
T^{(2)}(y)_{y \rightarrow \infty}=-C \frac{g^{2} A}{a \epsilon}\left(\ln \frac{c_{R}}{2 \epsilon}-C_{E}\right) .
$$

The ratio first to second order is at $y \rightarrow \infty$

$$
r^{(2)}(y)=\left.\frac{T^{(2)}(y)}{T^{(0)}(y)}\right|_{y>>1}=-\frac{\lambda^{2}}{4 \pi \alpha^{\prime} \epsilon}\left(\ln \frac{c_{R}}{2 \epsilon}-C_{E}\right) .
$$

Remarkably it does not depend on $A$.

\section{$5.33 \mathrm{~d}$ order}

In the third order the forward scattering amplitude is

$$
T^{(3)}(y)=\pi R_{A}^{2} \int d y_{2} d y_{3} G_{f}\left(y, y_{2}, 0\right) \Sigma_{2}\left(y_{2}, y_{3}, 0\right) T^{(0)}\left(y_{3}, b\right) .
$$

Using (63) at $k=0$ and interchanging the order of integration in $y_{2}$ and internal integration in $y_{1}$ inside $\Sigma_{2}$ we find

$$
T^{(3)}(y)=-g^{2} A \frac{\lambda^{2} a \epsilon}{2 \pi \alpha^{\prime}} \frac{e^{\epsilon y}}{p^{2}(y)} \int_{0}^{y} d y_{2} \frac{e^{\epsilon y_{2}}}{p^{2}\left(y_{2}\right)} \int_{0}^{y_{2}} \frac{d y_{1}}{y_{2}-y_{1}} p^{3}\left(y_{1}\right) e^{-\epsilon y_{1}} \int_{y_{1}}^{y_{2}} d y_{3} \frac{e^{\epsilon y_{3}}}{p\left(y_{3}\right)} .
$$


It is not difficult to find the asymptotic of $T^{(3)}(y)$ at large $y$. We present (92) in the form

$$
T^{(3)}(y)=-g^{2} A \frac{\lambda^{2} a \epsilon}{2 \pi \alpha^{\prime}} \frac{e^{\epsilon y}}{p^{2}(y)} \int_{0}^{y} d y_{2} \frac{e^{\epsilon y_{2}}}{p^{2}\left(y_{2}\right)} F\left(y_{2}\right),
$$

where taking $y_{1}=y_{2} \beta_{1}$ and $y_{3}=y_{2} \beta_{3}$

$$
F\left(y_{2}\right)=y_{2} \int_{0}^{1} \frac{d \beta_{1}}{1-\beta_{1}} p^{3}\left(y_{2} \beta_{1}\right) e^{-\epsilon y_{2} \beta_{1}} \int_{\beta_{1}}^{1} d \beta_{3} \frac{e^{\epsilon y_{2} \beta_{3}}}{p\left(y_{2} \beta_{3}\right)} .
$$

We are interested in the behaviour of $F\left(y_{2}\right)$ as $y_{2}>>1$, since this governs the behaviour of the integral over $y_{2}$ in (93). As $y_{2} \rightarrow \infty$ we have $p\left(y_{2} \beta\right) \rightarrow a \exp \left(\epsilon y_{2} \beta\right)$ so that

$$
F\left(y_{2}\right)_{y_{2}>>1}=a^{2} y_{2} \int_{0}^{1} \frac{d \beta_{1}}{1-\beta_{1}} e^{2 \epsilon y_{2} \beta_{1}} \int_{\beta_{1}}^{1} d \beta_{3}=\frac{a^{2}}{2 \epsilon}\left(e^{2 \epsilon y_{2}}-1\right) .
$$

Putting this asymptotic in (93) we obtain

$$
T^{(3)}(y)_{y>>1}=-g^{2} A \frac{\lambda^{2}}{4 \pi a \alpha^{\prime}} e^{-\epsilon y} \int_{0}^{y} d y_{2} e^{\epsilon y_{2}} \simeq-g^{2} A \frac{\lambda^{2}}{4 \pi a \alpha^{\prime} \epsilon}
$$

Recalling that at $y>>1 T^{(0)}=g^{2} A / a$ we find the ratio

$$
r^{(3)}(y)=\left.\frac{T^{(3)}(y)}{T^{(0)}(y)}\right|_{y>>1}=-\frac{\lambda^{2}}{4 \pi \alpha^{\prime} \epsilon}
$$

Note that in the particular case $a=1, T^{(3)}$ can easily be found explicitly at all $y$. In fact trivial integrations give

$$
T_{a=1}^{(3)}(y)=-g^{2} A \frac{\lambda^{2}}{4 \pi \alpha^{\prime} \epsilon}\left(1-e^{-\epsilon y}\right)^{2} .
$$

The full ratio $T / T^{(0)}$ from both loops, Fig. $2 a$ and $b$, turns out to be

$$
r(y)=r^{(2)}(y)+r^{(3)}(y)=-\frac{\lambda^{2}}{4 \pi \alpha^{\prime} \epsilon}\left(\ln \frac{c_{R}}{2 \epsilon}+1-C_{E}\right) .
$$

The bracket is universal. So one may define the renormalized self-mass by requiring that this bracket is zero, which implies that the loop correction vanishes at large $y$ :

$$
\ln \frac{c_{R}}{2 \epsilon}-C_{E}+1=0, \text { or } c_{R}=2 \epsilon e^{C_{E}-1} .
$$

This allows to experimentally determine $a$ and hence $\epsilon$ from the behaviour of the amplitude at large $y$. In this way we may define the renormalized intercept in a physically reasonable manner.

Note that with this choice of $c_{R}$ the renormalized intercept $\epsilon^{(r)}$ formally defined as the position of the pole of the propagator in the vacuum at $k=0$ is given by the solution of the equation (50) which reads

$$
\epsilon^{(r)}-\epsilon-C\left[\ln \frac{\epsilon^{(r)}-2 \epsilon}{2 \epsilon}+1\right]=0
$$

At small $\lambda$ it is complex and close to $\epsilon$. 


\subsection{Random phase approximation}

One may try to sum all primitive loop insertions into the amplitude ('random phase approximation'). In this approximation the amplitude $T(y)$ in the nuclear matter satisfies the Dyson equation

$$
T(y)=T^{(0)}(y)+\int_{0}^{y} d y_{1} \int_{0}^{y_{1}} d y_{2} G_{f}^{(0)}\left(y, y_{1}, 0\right)\left(\Sigma_{1}\left(y_{1}, y_{2}, 0\right)+\Sigma_{2}\left(y_{1}, y_{2}, 0\right)\right) T\left(y_{2}\right),
$$

where $\Sigma_{1}$ and $\Sigma_{2}$ are the 2 nd and 3 d order self-mass contributions studied above.

We present

$$
T(y)=T^{(0)}(y) r(y)
$$

The equation for $r(y)$ reads

$$
r(y)=1+X_{1}(y)+X_{2}(y)
$$

where $X_{1}$ and $X_{2}$ are parts coming from $\Sigma_{1}$ and $\Sigma_{2}$ respectively.

We find

$$
X_{1}(y)=-\frac{C}{p(y)} \ln \left(c_{R} y_{\text {min }}\right) \int_{0}^{y} d y_{1} p\left(y_{1}\right) r\left(y_{1}\right)-\frac{C}{p(y)} \int_{0}^{y} d y_{1} \int_{0}^{y_{1}} d y_{2} \frac{1}{y_{1}-y_{2}} e^{\epsilon\left(y_{1}-y_{2}\right)} \frac{p^{3}\left(y_{2}\right)}{p^{2}\left(y_{1}\right)} r\left(y_{2}\right) .
$$

Again we pass to variable $z=y_{1}-y_{2}$ to rewrite the last term as

$$
-\frac{C}{p(y)} \int_{0}^{y} \frac{d z}{z} \omega(y, z)=-\frac{C}{p(y)} \int_{0}^{y} \frac{d z}{z}(\omega(y, z)-\omega(y, 0))-\frac{C}{p(y)} \omega(y, 0) \int_{y_{\min }}^{y} \frac{d z}{z},
$$

where

$$
\omega(y, z)=e^{\epsilon z} \int_{z}^{y} d y_{1} \frac{p^{3}\left(y_{1}-z\right)}{p^{2}\left(y_{1}\right)} r\left(y_{1}-z\right) .
$$

Obviously

$$
\omega(y, 0)=\int_{0}^{y} d y_{1} p\left(y_{1}\right) r\left(y_{1}\right)
$$

so that the last term in (106) changes $y_{\min }$ in (105) to $y$. As a result we find that the part $X_{1}(y)$ in the equation for $r$ takes the form

$$
X_{1}=-\frac{C}{p(y)} \ln \left(c_{R} y\right) \int_{0}^{y} d y_{1} p\left(y_{1}\right) r\left(y_{1}\right)-\frac{C}{p(y)} \int_{0}^{y} \frac{d z}{z}(\omega(y, z)-\omega(y, 0)) .
$$

The part $X_{2}(y)$ is

$$
X_{2}(y)=-\frac{2 a \epsilon C}{p(y)} \int_{0}^{y} d y_{1} \frac{e^{\epsilon y_{1}}}{p^{2}\left(y_{1}\right)} \int_{0}^{y_{1}} \frac{d y_{3}}{y_{1}-y_{3}} p^{3}\left(y_{3}\right) e^{-\epsilon y_{3}} \int_{y_{3}}^{y_{1}} d y_{2} \frac{e^{\epsilon y_{2}}}{p\left(y_{2}\right)} r\left(y_{2}\right) .
$$

In the case $a=1$ and neglecting $X_{2}$ we can solve the Dyson equation passing to the Laplace transforms in rapidity. We find

$$
T(E)=\frac{T^{(0)}(E)\left(G_{f}^{(0)}\right)^{-1}(E, 0)}{\left(G_{f}^{(0)}\right)^{-1}(E, 0)-\Sigma_{f}^{(r)}(E, 0)}
$$

where $G_{f}^{(0)}(E, 0)$ and $\Sigma_{f}^{(r)}(E, 0)$ have the same form as (47) and (48) with the opposite sign of $\epsilon$ and

$$
T^{(0)}(E)=g^{2} A \frac{1}{E} \text {. }
$$


So the Laplace transform for the amplitude is

$$
T(E)=\frac{g^{2} A}{E} \frac{E+\epsilon}{E+\epsilon-C\left[\ln (E+2 \epsilon)+C_{E}-\ln c_{R}\right]} .
$$

From the expression for $T(E)$ we immediately conclude that in the limit $y \rightarrow \infty$ the amplitude tends to

$$
\left.T(y)\right|_{y \rightarrow \infty}=g^{2} A Z
$$

where

$$
Z=\frac{\epsilon}{\epsilon-C\left[\ln (2 \epsilon)+C_{E}-\ln c_{R}\right]} .
$$

Factor $Z-1$ gives the correction from loops. We can define the renormalization condition by requiring that $Z=1$, which implies for this case $\left(X_{2}=0\right)$

$$
\ln (2 \epsilon)+C_{E}-\ln c_{R}=0
$$

With this choice

$$
\Sigma^{(r)}(E, k)=C \ln \frac{E+2 \epsilon+\alpha^{\prime} k^{2} / 2}{2 \epsilon}
$$

and

$$
T(E)=\frac{g^{2} A}{E} \frac{E+\epsilon}{E+\epsilon-C \ln \frac{E+2 \epsilon}{2 \epsilon}} .
$$

\section{Pomeron Green function in the random phase approximation}

In this approximation the full Green function in the nuclear matter $G_{f}(y, 0, k)$ satisfies the Dyson equation

$$
G_{f}(y, 0, k)=G_{f}^{(0)}(y, 0, k)+\int_{0}^{y} d y_{1} \int_{0}^{y_{1}} d y_{2} G_{f}^{(0)}\left(y, y_{1}, k\right)\left(\Sigma_{1}\left(y_{1}, y_{2}, k\right)+\Sigma_{2}\left(y_{1}, y_{2}, k\right)\right) G_{f}\left(y_{2}, 0, k\right)
$$

We present

$$
G_{f}(y, 0, k)=G_{f}^{(0)}(y, 0, k) R(y, k)
$$

to obtain an equation for $R$

$$
R(y, k)=1+Y_{1}+Y_{2}
$$

Here $Y_{1}$ comes from $\Sigma_{1}$ :

$$
Y_{1}(y, k)=-C \ln \left(c_{R} y_{\min }\right) \int_{0}^{y} d y_{1} R\left(y_{1}, k\right)-C \int_{0}^{y} d y_{1} \int_{0}^{y_{1}} d y_{2} \frac{1}{y_{1}-y_{2}} e^{\beta\left(y_{1}-y_{2}\right)} \frac{p^{2}\left(y_{2}\right)}{p^{2}\left(y_{1}\right)} R\left(y_{2}, k\right)
$$

and $\beta=\epsilon+\alpha^{\prime} k^{2} / 2$. Passing to integration variable $z=y_{1}-y_{2}$ we rewrite the last term in Eq. (122) as

$$
-C \int_{0}^{y} \frac{d z}{z} \Omega(y, z)=-C \int_{0}^{y} \frac{d z}{z}(\Omega(y, z)-\Omega(y, 0))-C \Omega(y, 0) \int_{y_{\min }}^{y} \frac{d z}{z}
$$

where

$$
\Omega(y, z)=e^{\beta z} \int_{z}^{y} d y_{1} \frac{p^{2}\left(y_{1}-z\right)}{p^{2}\left(y_{1}\right)} R\left(y_{1}-z, k\right) .
$$


Obviously

$$
\Omega(y, 0)=\int_{0}^{y} d y_{1} R\left(y_{1}, k\right),
$$

so that the last term in (123) changes $y_{\min }$ in (122) to $y$. As a result we find

$$
Y_{1}(y, k)=-C \ln \left(c_{R} y\right) \int_{0}^{y} d y_{1} R\left(y_{1}, k\right)-C \int_{0}^{y} \frac{d z}{z}(\Omega(y, z)-\Omega(y, 0)) .
$$

The part $Y_{2}$ comes from $\Sigma_{2}$ :

$$
\begin{gathered}
Y_{2}(y, k)= \\
-2 a \epsilon C \int_{0}^{y} d y_{1} \frac{e^{\epsilon y_{1}}}{p^{2}\left(y_{1}\right)} \int_{0}^{y_{1}} \frac{d y_{2}}{y_{1}-y_{2}} p^{3}\left(y_{2}\right) e^{-\epsilon y_{2}} \int_{y_{2}}^{y_{1}} d y_{3} \frac{e^{\epsilon y_{3}}}{p^{2}\left(y_{3}\right)} R\left(y_{3}, k\right) \exp \left(\frac{1}{2} \alpha^{\prime} k^{2} \frac{\left(y_{1}-y_{3}\right)^{2}}{y_{1}-y_{2}}\right) .
\end{gathered}
$$

In the general case Eq. (126) can only be solved numerically. In a particular case $a=1$ both the Green function $G^{(0)}$ and $\Sigma_{1}$ depend only on the rapidity difference:

$$
p(y)=e^{\epsilon y}
$$

and

$$
\begin{gathered}
G_{f}^{(0)}\left(y_{1}, y_{2}, k\right)=e^{-\left(y_{1}-y_{2}\right)\left(\epsilon+\alpha^{\prime} k^{2}\right)}=G_{f}^{(0)}\left(y_{1}-y_{2}, k\right) \\
\Sigma_{1}\left(y_{1}, y_{2}, k\right)=-C\left(\frac{1}{y_{1}-y_{2}} e^{-\left(y_{1}-y_{2}\right)\left(2 \epsilon+\alpha^{\prime} k^{2} / 2\right)}+\delta\left(y_{1}-y_{2}\right) \ln \left(c_{R} y_{m i n}\right)=\Sigma_{1}\left(y_{1}-y_{2}, k\right)\right.
\end{gathered}
$$

They have the same form as in the vacuum with the opposite sign of $\epsilon$. So if one additionally neglects the formally $3 \mathrm{~d}$-order contribution $\Sigma_{2}$ the Dyson equation (119) can be again analytically solved by the Laplace transform in the same way as in the vacuum case. Moreover, the solution is exactly the same as in the vacuum case with the change $\epsilon \rightarrow-\epsilon$ :

$$
G_{f}^{-1}(E, k)=E+\epsilon+\alpha^{\prime} k^{2}-C\left[\ln \left(E+2 \epsilon+\alpha^{\prime} k^{2} / 2\right)+C_{E}-\ln c_{R}\right] .
$$

Singularities of $G_{f}(E, k)$ in the complex $E$ - plane consist of a left cut starting at $E_{c}=-2 \epsilon-\alpha^{\prime} k^{2} / 2$ and corresponding to the standard cut generated by a subcritical pomeron with intercept $1-\epsilon$ and a possible pole whose position depends on the renormalization constant $c_{R}$ and which determines the asymptotic at large rapidities. With the choice (116) corresponding to $\Sigma_{2}=0$ we have

$$
G_{f}^{-1}(E, k)=E+\epsilon+\alpha^{\prime} k^{2}-C \ln \frac{E+2 \epsilon+\alpha^{\prime} k^{2} / 2}{2 \epsilon}
$$

The Green function vanishes at $y \rightarrow \infty$ as $e^{-y \epsilon_{0}}$ where for $\alpha^{\prime} k^{2}<2 \epsilon$

$$
\epsilon+\alpha^{\prime} k^{2}<\epsilon_{0} \leq 2 \epsilon+\alpha^{\prime} k^{2} / 2 .
$$

If $\alpha^{\prime} k^{2} \geq 2 \epsilon$, the two-pomeron cut moves to the right of the pole, which splits into two complex conjugate poles on the physical sheet, and the asymptotic is determined by the cut so that $y_{0}=$ $2 \epsilon+\alpha^{\prime} k^{2} / 2$ imaginary parts of the two terms in the denominator are opposite.

Unfortunately inclusion of $\Sigma_{2}$ spoils invariance with respect to translations in rapidity, and solution of the Dyson equation by means of the Laplace transform becomes impossible. 


\section{$7 \quad$ Numerical illustration}

In this section we report on numerical results for the amplitude $T(y)$ and Green function $G_{f}(y, k)$ which follow from the Dyson equations (102) and (119) with the pomeron self-mass $\Sigma=\Sigma_{1}+\Sigma_{2}$ given by loops of Fig. $2 a$ and $b$ and realistic values of $\epsilon, \alpha^{\prime}, \lambda$ and $g$ For the latter we take the standard values, which correspond to the experimental data at comparatively low energies

$$
\epsilon=0.08, \quad \alpha^{\prime}=0.2 \quad \mathrm{GeV}^{-2}, \quad \lambda=-0.48 \mathrm{GeV}^{-1}, \quad g=5.94 \mathrm{GeV}^{-1}
$$

For the transverse nuclear density we choose that in the center of the nucleus with the constant nuclear density within a sphere of radius $R_{A}=A^{1 / 3} \cdot 1.15 \mathrm{fm}$.

With $\epsilon, \alpha^{\prime}$ and $\lambda$ given by (134) we obtain from Eq. (101) the renormalized values for the intercept and slope in the vacuum:

$$
\epsilon^{(r)}=0.155 \pm i 0.139, \quad \alpha^{\prime(r)}=0.172 \mp i 0.043
$$

Both are complex. The real part of the pomeron trajectory is found to be linear in $k^{2}$ with a good precision. The imaginary part is not, the value of $\operatorname{Im} \alpha^{\prime(r)}$ diminishing from 0.043 at $k=0$ to 0.020 at $k=2 \mathrm{GeV} / \mathrm{c}$. So we are dealing with a supercritical pomeron with a complex intercept and slope.

Next we pass to the amplitude and pomeron Green function in the nuclear background.

Fig. 4 shows the ratio $r(y)$ of the calculated amplitude to the lowest order one as a function of $y$ for two values of the atomic number $A=64$ and 207. As one observes as rapidity grows the ratio initially goes up reaching at its maximum values of the order $2.2 \div 2.3$ depending on $A$ and then gradually goes to unity, as determined by the chosen value of $c_{R}$. The $A$-dependence is in fact weak, so that the two curves are close to one another. The rather large values of the ratio at intermediate rapidities show that for the chosen parameters the loop contribution is not at all small. In other words the strength of the triple pomeron interaction is relatively large although it effectively goes down at large rapidities.

In Figs. 5, 7 we show the $R(y, k)$ of the Green function to its lowest order value as a function of $y$ for values of $k=0,1$ and $2 \mathrm{GeV} / \mathrm{c}$ and $A=64$ and 207. For convenience for $k=2 \mathrm{GeV} / \mathrm{c}$

we plot $R_{1}(y, k)=-R(y, k) \exp \left(y\left(\epsilon-\alpha^{\prime} k^{2} / 2\right)\right)$, since for such large $k$ the asymptotic is negative and governed by the cut contribution. Again we observe that the $A$-dependence is weak. The $y$-dependence strongly depends on the chosen value of $k$, which is to be expected, since the cut contribution vanishes at $k \rightarrow \infty$ much slower, with a twice smaller slope. For all momenta the loop contribution is relatively large at all rapidities, which again indicates that with our choice $\lambda$ is not small. Oscillations observed in Figs. [5 and 6] illustrate that the 'physical' intercept $\epsilon^{(r)}$ for moderate $k$ in fact splits into two complex conjugate values. At larger $k$ the asymptotic is taken over by the cut contribution and oscillations disappear.

\section{Conclusions}

We studied the contributions of the two simplest loops in the local reggeon field theory with a supercritical pomeron in the nucleus. Our results show that the nuclear surrounding effectively transforms the supercritical pomeron with the intercept $\alpha(0)-1=\epsilon>0$ into the subcritical one with the intercept $-\epsilon$. As a result, at high energies the pomeron Green function vanishes and 
contributions from multipomeron exchanges vanish still faster according to the standard predictions for the subcritical pomeron. With that, for a finite nucleus with a constant profile function, the scattering amplitude tends to a constant value at high energies. Contribution from self-mass insertions do not change this behaviour, since in the rapidity space they are dominated by the configuration in which these insertions enter at small rapidities and the bulk of the rapidity is covered by the lowest order amplitude. With an appropriate choice of the renormalization constant one can make the contribution from loops vanish at high energies. The renormalized pomeron intercept in this theory (in vacuum) is complex with a positive real part. This makes the conventional renormalization technique inapplicable.

These results have been obtained for a simple case of a constant nuclear profile function $T(b)$ and with only lowest order loops taken into account. The limitation to a constant $T(b)$ is technical and we believe that the same conclusions can be obtained for a realistic $T(b)$. However this requires using non-trivial pomeron Green functions in the external $b$-dependent field, which can only be calculated numerically. Construction of loops in this case presents a formidable calculational problem, which seems to be outside our technical possibilities. The only difference we expect with a realistic $T(b)$ is due to its long distance tail. Then at large $b>b_{0}(y)$ the amplitude and the Green function will no more be damped by the nucleus and become purely perturbative, that is growing as $e^{\epsilon y}$ at large $y$. Very crudely we expect $b_{0}$ to be determined by the relation $T\left(b_{0}\right) e^{\epsilon y} \sim 1$ which gives $b_{0}(y) \sim a y$ where $a$ is an $A$-independent parameter of the dimension of length $(a=0.545 \mathrm{fm}$ with the Woods-Saxon nucleus density). Then, again very crudely, the cross-section obtained by the integration over all $b$ will become proportional to $y^{2}$ in accordance with the conclusions in [11]. Inclusion of more complicated loops does not present any difficulty of principle either. For small enough $\lambda$ their contribution will be small at all rapidities and we do not expect any qualitative change.

Note that with the conventional values (134) for $\epsilon, \alpha^{\prime}$ and $\lambda$ the loop contribution proves to be not at all small, in spite of the rather small value of the effective loop parameter $C \sim 0.09$. The renormalized intercept (135) obtained as the solution of Eq.(50) is quite different from its zeroorder value $\epsilon=0.08$, which testifies that the coupling constant $\lambda$ is in fact quite large. Because of that we cannot claim that with the conventional parameters (134) our results are complete . More complicated loops have to be included to make the results reliable and fit to be used for the description of physical observables.

This is unfortunate, but we consider our results to be interesting mostly from the purely theoretical point of view. They may serve as a basis for treating loop contributions in the perturbative QCD. It seems to be advantageous to study them in the nuclear surrounding, which makes the high-energy behaviour much more tractable.

\section{Appendix A: Possible poles of propagators}

Let the denominator in (49) be

$$
D(E, k)=E-\epsilon+\alpha^{\prime} k^{2}-|C|\left[\ln \left(E-2 \epsilon+\alpha^{\prime} k^{2} / 2\right)+C_{E}-\ln c_{R}\right],
$$

where we have taken into account that $C<0$. We denote $\delta=|C|\left(C_{E}-\ln c_{R}\right)$ and rewrite (A1) as

$$
D(E, k)=E-\epsilon+\alpha^{\prime} k^{2}-\delta-|C| \ln \left(E-2 \epsilon+\alpha^{\prime} k^{2} / 2\right) .
$$

Function $D(E, k)$ has a left cut starting at $E=2 \epsilon-\alpha^{\prime} k^{2} / 2$, so that it can vanish only to the right of the branchpoint. At $E \rightarrow 2 \epsilon-\alpha^{\prime} k^{2} / 2$ from the right $D(E, k) \rightarrow+\infty$. At $E \rightarrow+\infty$ also 
$D(E, k) \rightarrow+\infty$. The single minimum of $D(E, k)$ as a function of $E$ occurs at

$$
\frac{\partial D(E, k)}{\partial E}=1-|C| \frac{1}{E-2 \epsilon+\alpha^{\prime} k^{2} / 2}=0,
$$

that is at

$$
E_{\text {min }}=e^{|C|}+2 \epsilon-\frac{1}{2} \alpha^{\prime} k^{2}
$$

The minimal value of $D(E, k)$ is

$$
D_{\min }(E, k)=e^{|C|}+\epsilon+\frac{1}{2} \alpha^{\prime} k^{2}-\delta-|C|^{2} .
$$

Obviously if $D_{\min }>0$ then $D(E, k)$ does not vanish on the physical sheet of $E$. If $D_{\min }<0$ there are two zeros to the right of the cut. The rightmost zero $E_{0}$ determines the asymptotic of the propagator at large $y$. The value of $D_{\min }$ depends on the choice of $\delta$. So if

$$
\delta>e^{|C|}+\epsilon+\frac{1}{2} \alpha^{\prime} k^{2}-|C|^{2}
$$

the propagator develops two poles to the right of the branchpoint at $E=2 \epsilon-\alpha^{\prime} k^{2} / 2$, the larger of which is located to the right of $E_{\min }$ and thus to the right of the unperturbed pole at $E=\mu-\alpha^{\prime} k^{2}$. This larger pole takes the function of the renormalized intercept. If, on the other hand, relation (A6) is not satisfied, the propagator has no poles on the physical sheet and its asymptotic at $y \rightarrow \infty$ is determined by the branchpoint at $E=2 \epsilon-\alpha^{\prime} k^{2} / 2$

Passing to the nuclear surrounding with $a=1$, at $k=0$ we find a denominator

$$
D(E)=E+\epsilon-|C| \ln \frac{E+2 \epsilon}{2 \epsilon}
$$

We put $E+2 \epsilon=2 \epsilon \xi,|C| /(2 \epsilon)=\eta<<1$, so that zeros are determined by the equation for $\xi$

$$
f(\xi)=\xi-\eta \ln \xi-\frac{1}{2}=0 .
$$

The minimum of $f(\xi)$ occurs at $\xi_{\text {min }}=\eta$ and

$$
f_{\min }=\eta-\eta \ln \eta-\frac{1}{2}
$$

At $\eta<1 f_{\min }<0$ for $\eta<0.186683$, for which values there exist two zeros

$$
0<\xi_{1}<\eta<\xi_{2}<\frac{1}{2}
$$

(the limiting value $1 / 2$ is achieved by the larger zero at $\eta \rightarrow 0$ ). This means that poles in the propagator exist for small enough $\eta$ and are located between the unperturbed pole at $E=-\epsilon$ and branchpoint at $E=2 \epsilon$. One can easily see that this result is also true for $k^{2}>0$. So the poles do not change the asymptotic of the amplitude, but diminish the intercept of the propagator, making it still more subcritical. 


\section{References}

[1] I.I.Balitsky, Nucl. Phys. B 463, 99 (1996)

[2] Yu.V.Kovchegov, Phys. Rev. D 60, 034008 (1999); 61, 074018 (2000)

[3] M.A.Braun, Eur. Phys. J. C 16, 337 (2000)

[4] A.Schwimmer, Nucl.Phys. B 94, 445 (1975)

[5] D.Amati, L.Caneschi, R.Jengo, Nucl. Phys. 101, 397 (1975)

[6] V.Alessandrini, D.Amati, R.Jengo, Nucl.Phys. B 108, 425 (1976)

[7] R.Jengo, Nucl. Phys. B 108, 425 (1976)

[8] M.Ciafaloni, M.Le Bellac, G.C.Rossi, Nucl. Phys. B 130, 388 (1977)

[9] M.A.Braun, G.P.Vacca, Eur. phys. J. C 50, 857 (2007)

[10] H.D.Abarbanel, J.B.Bronzan, A.Schwimmer, R.Sugar, Phys. Rev. D 14, 632 (1976)

[11] D.Amati, M.Le Bellac, G.Marchesini, M.Ciafaloni, Nucl. Phys. B 112, 107 (1976)

\section{Figure captions}

Figure 1: The new vertex for two-pomeron annihilation, which appears after the shift in field $\phi^{\dagger}$. Figure 2: Some simple loop diagrams for the pomeron Green function.

Figure 3: Diagrams with one loop ( $a, b$ and two loops $(c$ and $d)$ for the scattering amplitude.

Figure 4: The ratio $r(y)$ of forward scattering amplitude to its lowest order for $\mathrm{Cu}$ and $\mathrm{Pb}$ targets. Figure 5: The ratio $R(y)$ of the pomeron Green function to its lowest order at $k=0$ for $\mathrm{Cu}$ and $\mathrm{Pb}$ targets.

Figure 6: Same as Fig. 5 at $k=1 \mathrm{GeV} / \mathrm{c}$.

Figure 7: The scaled ratio $R_{1}(y, k)=-R(y, k) \exp \left(y\left(\epsilon-\alpha^{\prime} k^{2} / 2\right)\right)$ at $k=2 \mathrm{GeV} / \mathrm{c}$ for $\mathrm{Cu}$ and $\mathrm{Pb}$ targets. 


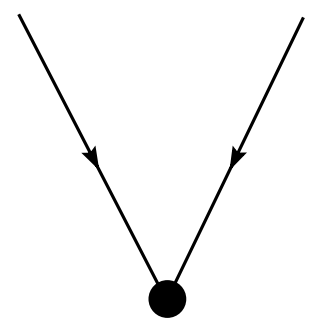

Figure 1: The new vertex for two-pomeron annihilation, which appears after the shift in field $\phi^{\dagger}$

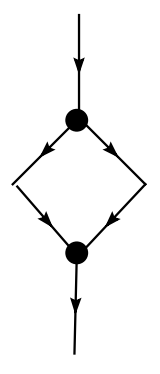

a

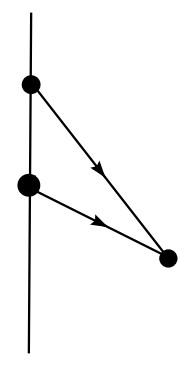

$b$

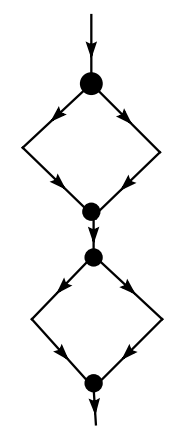

$c$

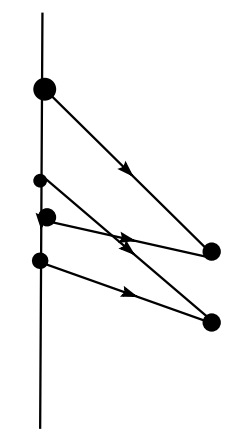

$d$

Figure 2: Some simple loop diagrams for the pomeron Green function

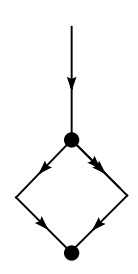

a

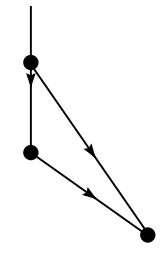

$b$

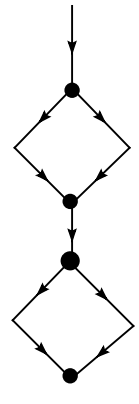

$c$

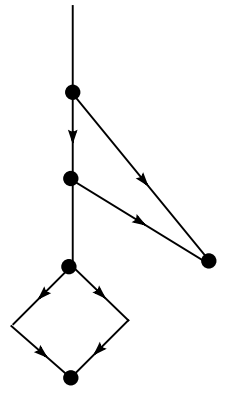

$d$

Figure 3: Diagrams with one loop ( $a, b$ and two loops $(c$ and $d)$ for the scattering amplitude 


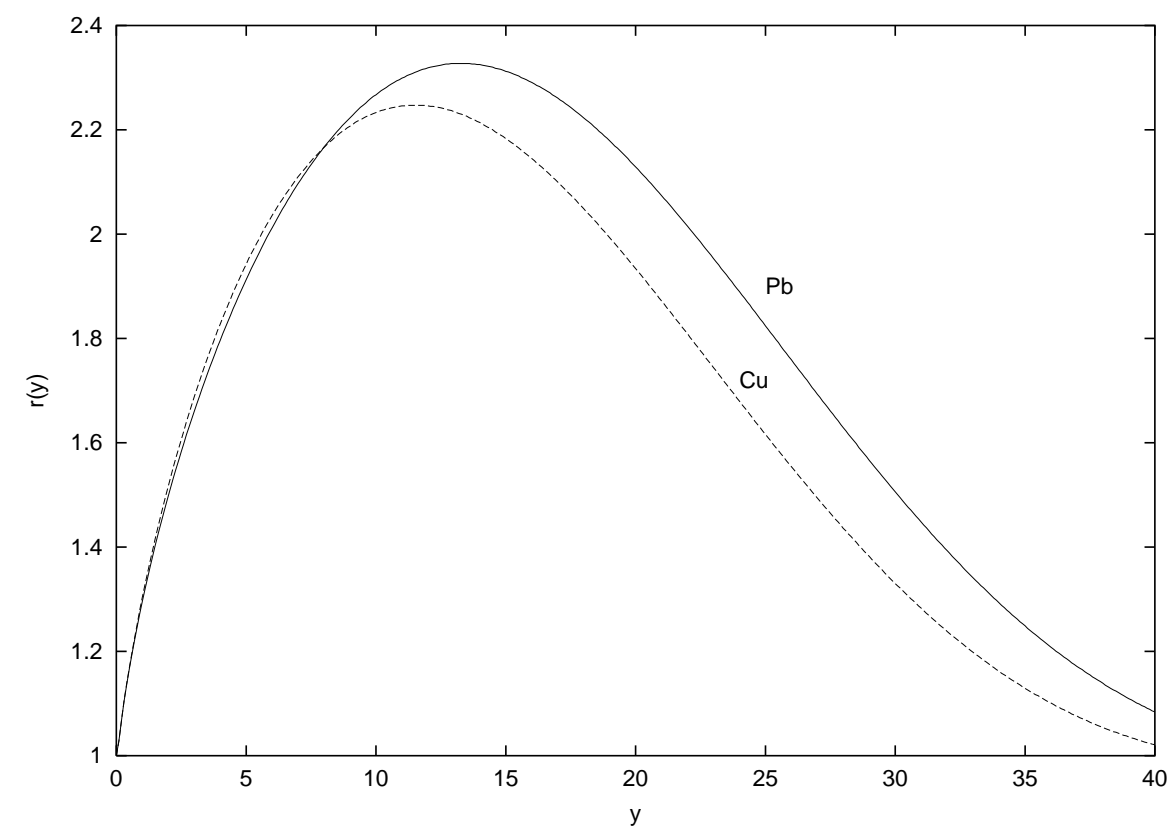

Figure 4: The ratio $r(y)$ of forward scattering amplitude to its lowest order for $\mathrm{Cu}$ and $\mathrm{Pb}$ targets

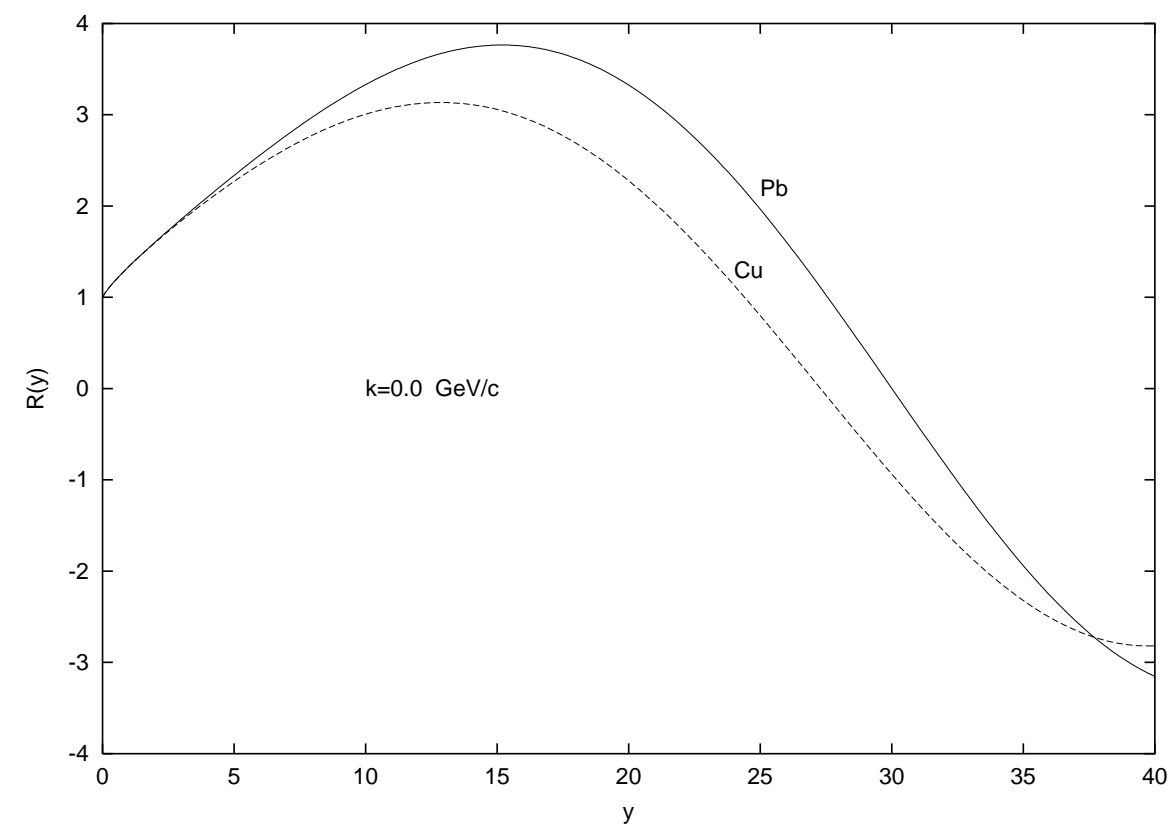

Figure 5: The ratio $R(y)$ of the pomeron Green function to its lowest order at $k=0$ for $\mathrm{Cu}$ and $\mathrm{Pb}$ targets 


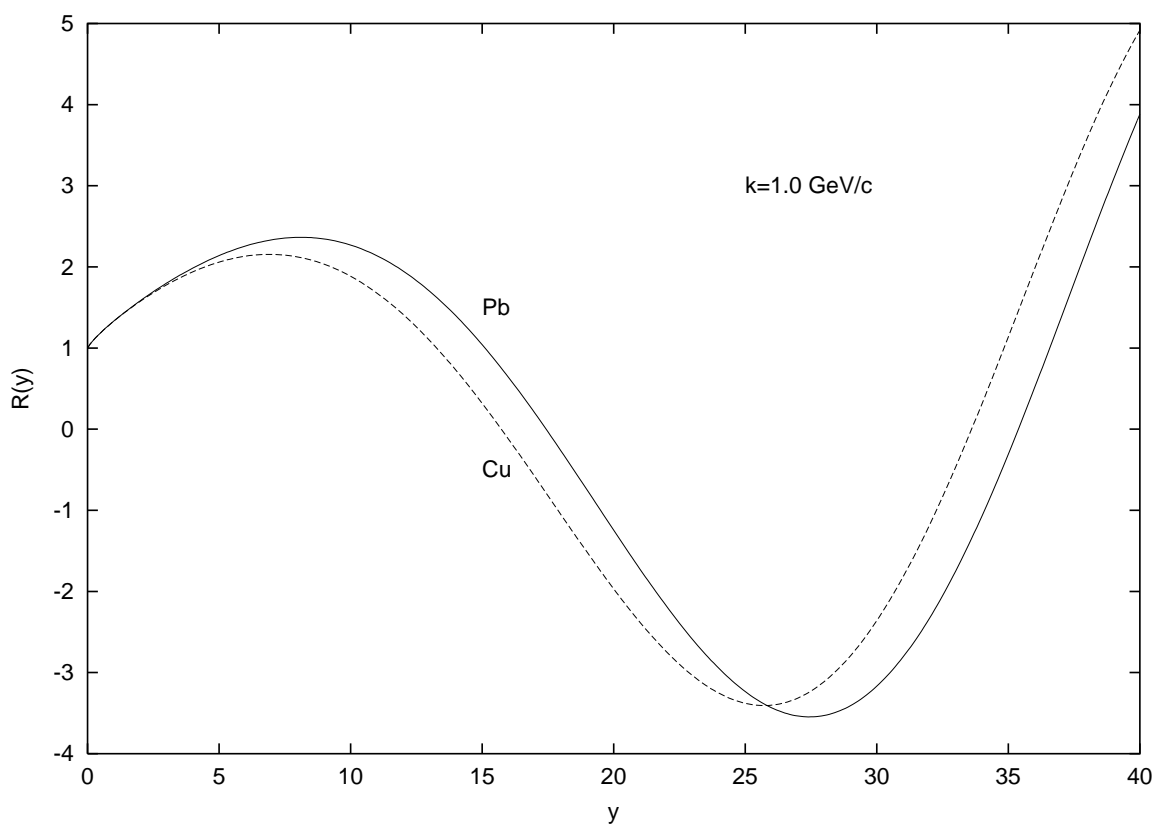

Figure 6: Same as Fig. 5 at $k=1 \mathrm{GeV} / \mathrm{c}$

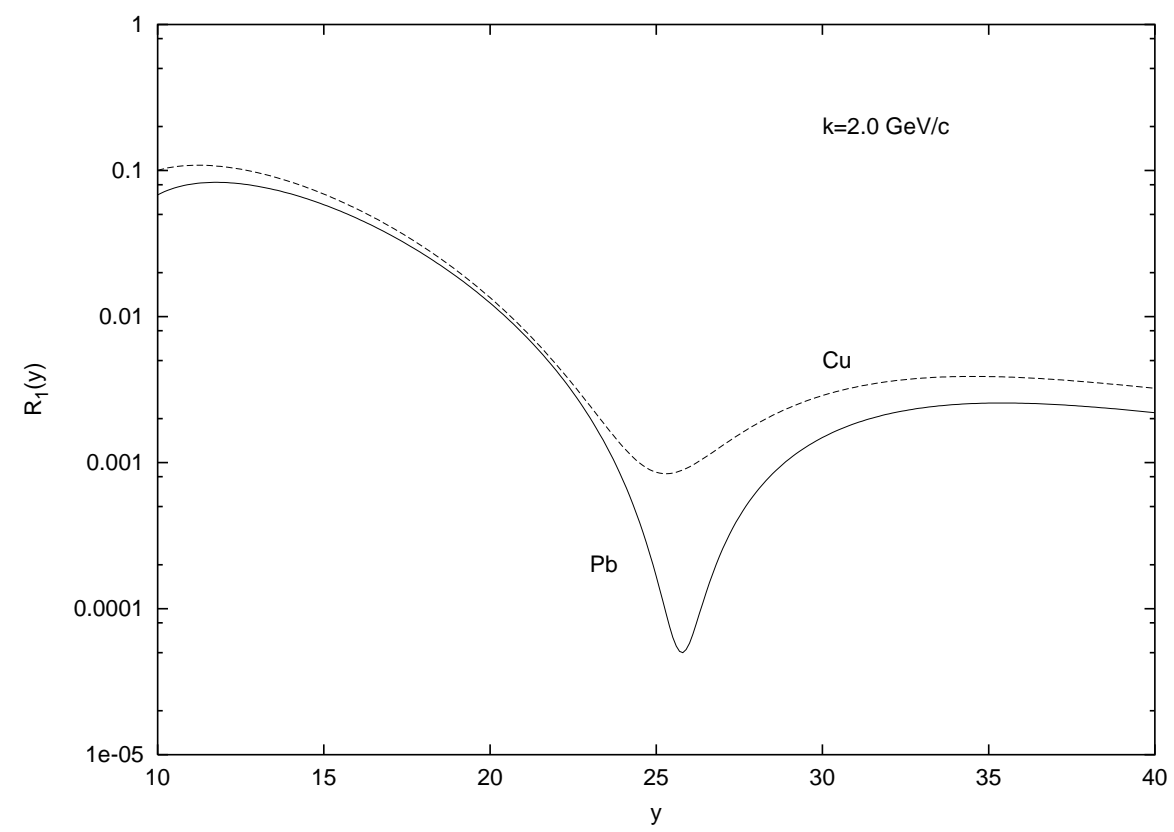

Figure 7: The scaled ratio $R_{1}(y, k)=-R(y, k) \exp \left(y\left(\epsilon-\alpha^{\prime} k^{2} / 2\right)\right)$ at $k=2 \mathrm{GeV} / \mathrm{c}$ for $\mathrm{Cu}$ and $\mathrm{Pb}$ targets 